\title{
Lipid metabolism does not influence the expression of proximal aortopathy in bicuspid aortic valve disease
}

\author{
Tatiana M Sequeira Gross', Thomas Kuntze, Evaldas Girdauskas \\ From World Society of Cardiothoracic Surgeons 25th Anniversary Congress, Edinburgh \\ Edinburgh, UK. 19-22 September 2015
}

\section{Background/Introduction}

Controversy exists regarding the pathogenesis of bicuspid aortic valve (BAV) associated aortopathy. Recent data indicate the potential role of lipid metabolism in the expression of aortopathy.

\section{Aims/Objectives}

We aimed to correlate the markers of lipid metabolism with severity of proximal aortopathy in patients with BAV vs. tricuspid aortic valve (TAV) disease.

\section{Method}

A total of 458 consecutive patients (mean age $64 \pm 11$ years, $68 \%$ male) underwent aortic valve replacement (AVR) with/without proximal aortic surgery from January,2008 through December,2014. All patients undergoing combined procedures (e.g., $A V R+C A B G$ ) were excluded. Only patients in whom proximal aortic dimensions were defined by preoperative CT/MRI and/or TOE were included. Correlation analysis was performed between markers of lipid metabolism (i.e., cholesterol, LDL, HDL, and triglyceride) and maximal diameter of the proximal aorta in BAV subgroup $(\mathrm{n}=273)$ vs. TAV subgroup $(n=185)$. Moreover, we compared correlation patterns between markers of lipid metabolism and maximal aortic diameter in BAV insufficiency $(n=46)$ vs. BAV stenosis $(n=227)$ cohorts. Logistic regression was performed to identify risk factors for proximal aortic diameter $>40 \mathrm{~mm}$ in BAV and TAV subgroups.

\section{Results}

No correlation was found between markers of lipid metabolism and proximal aortic diameter in BAV subgroup $(\mathrm{r}=-0.1, \mathrm{p}=0.1)$ and TAV subgroup $(\mathrm{r}=0.006, \mathrm{p}=0.9)$. No significant differences in correlation patterns were found between markers of lipid metabolism and maximal aortic diameter in BAV insufficiency $(\mathrm{r}=0.03, \mathrm{p}=0.8)$ vs. BAV stenosis $(r=-0.1, p=0.1)$ cohorts. Logistic regression analysis revealed triglyceride levels (HR 1.4, $\mathrm{p}=0.05)$ and statin therapy (HR $0.4, \mathrm{p}=0.03$ ) as predictors of proximal aortic diameter $>40 \mathrm{~mm}$ in TAV subgroup only.

\section{Discussion/Conclusion}

Our study demonstrates no linear correlation between markers of lipid metabolism and proximal aortic diameters in a surgical cohort of BAV and TAV patients. Statin therapy and triglyceride levels influence significantly proximal aortic diameter in patients with TAV, but not with BAV disease.

Published: 16 December 2015

\footnotetext{
doi:10.1186/1749-8090-10-S1-A65

Cite this article as: Gross et al:: Lipid metabolism does not influence the expression of proximal aortopathy in bicuspid aortic valve disease. Journal of Cardiothoracic Surgery 2015 10(Suppl 1):A65.
}

Department of Cardiac Surgery, Central Clinic Bad Berka, Germany 